\title{
UAV path planning research under the environment of moved target
}

\author{
Xia Chen ${ }^{1}$, Xingchao $\mathrm{Yu}^{1}$, Xianwei $\mathrm{Hu}^{2}$
}

1. School of Automation, Shenyang Aerospace University, China

2. Department of chemical process equipment, Shenyang Chemical Industry School, China

*xiachen1108@163.com; *yuxingchao2010@163.com; *wolf63@126.com

Keywords: UAV; path planning; Voronoi diagram; moved target; RRT algorithm

\begin{abstract}
In this paper, the problem of path planning existing target movement in the flight is studied. Firstly, according to the terrain threat, the threat field is created by using the Voronoi diagram method, and the total cost of the track is established. Then, the problem of the traditional path planning only meeting the fixed target is studied, and the method of using Rapidly-exploring Random Tree (RRT) algorithm to solve the problem of target movement is proposed, the path after the target moved is modified, the precise strike for the moved target is realized. The simulation results show that initial path planned by Voronoi diagram and modified path planned by RRT algorithm after finding the target moved can effectively solve the accurate strike problem for the moved target.
\end{abstract}

\section{Introduction}

Path planning as an important part of UAV mission planning system, not only improves the operational effectiveness of UAV and it is the effective means of long-range precision strike, but also it is the technical support to achieve UAV self-control and intelligent flight. Voronoi diagram can change complex path search problem in space area into a simple weighted graph search problem, thus the computational quantity could be reduced enormously ${ }^{[1]}$, RRT algorithm ${ }^{[2]}$ as a kind of fast search method, it has been widely used in the field of path planning research, especially in high dimensional space, the advantage of speed is especially obvious ${ }^{[3]}$. Particularly, in the face of unexpected situations can better respond. At present, the research of the static path planning is relatively mature, but the situation of target state information always changes in the real battlefield environment, therefore, it is more realistic to solve the problem of path planning for moving target ${ }^{[4]}$. In this paper, UAV path planning method under the environment of moved target is proposed based on Voronoi diagram-RRT algorithm.

\section{The description of path planning space based on Voronoi diagram}

The environment description of path planning based on Voronoi diagram is that regard sources of threats like enemy radar location as the generatrix $p=\left\{p_{1}, p_{2}, p_{3}, \ldots, p_{n}\right\}$ in the Voronoi diagram, the edges of Voronoi diagram are composed of the vertical of any adjacent parent point, which describe the planning environment. fig 1 shows the description of 14-point threat based on the planning environment of Voronoi diagram. Among them: $p_{1}, \ldots, p_{n}$ were threat points, black dotted line was the connection line between each threat point; black thin solid line was the edge of Voronoi diagram, which was expressed as flight path of the minimum level of threat between two threat points; the intersection of each side in Voronoi diagram was regarded as navigation point of aircraft flight path point; $S$ was the starting point of aircraft; target $T$ was the target point needed to be attacked; black thick solid line was feasible track from the starting point $\mathrm{S}$ to the target point $\mathrm{T}$. 


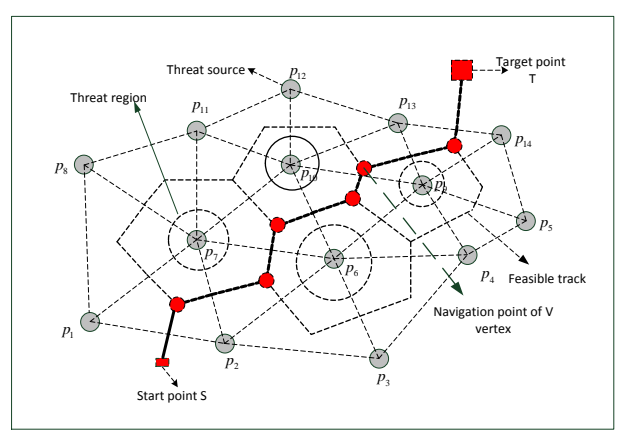

Fig 1. The description of path planning space based on the Voronoi diagram

\section{The function model of path planning based on Voronoi diagram}

The function model of path planning based on Voronoi diagram mainly included the following two aspects: fuel cost and radar threats cost.

3.1 The function model of fuel cost. When UAVs fly along each side of Voronoi diagram, the voyage of UAV is the sum of a straight line distance between each steering node, $H$ represents the fuel cost, so the function of fuel cost is:

$$
H=\sum_{a=1}^{N} l_{a}
$$

In formula: $N$ represents the number of voyage segments, $l_{a}$ represents the length of $a$-th segment voyage.

3.2 The function model of radar threat cost. Due to the intensity of the reflected radar echo is inversely proportional to the fourth power of distance to radar, so the threat cost of UAV flight along the Voronoi diagram edge can be considered to be the integration of radar threat at the edge of Voronoi diagram. In order to simplify the calculations, each edge of Voronoi can be used to discrete calculation, the distance of taking $1 / 6,1 / 2$ and $5 / 6$ of each edge to the each threat can be used to calculating the value of threat cost. For the $a$-th edge, the radar threat cost can be expressed as:

$$
J_{a}=l_{a} \sum_{b=1}^{M}\left(\frac{1}{d_{1 / 6, a, b}^{4}}+\frac{1}{d_{1 / 2, a, \mathrm{~b}}^{4}}+\frac{1}{d_{5 / 6, \mathrm{a}, \mathrm{b}}^{4}}\right)
$$

In formula: $M$ represents the number of radar in the threats field, $l_{a}$ represents the length of $a$-th segment voyage; $d$ represents the distance from the threat to the $a$-th path. Note: every radar has a strong role scope, when the UAV flys to this range, the UAV would be detected and destroyed with the probability of one hundred percent. Therefore, we must avoid the radar. Assuming its scope is a circle, the radar is center of the circle, $r_{0}$ is the radius, if the distance from the $a$-th edge of Voronoi diagram to the radar point is $d_{\mathrm{a}, \mathrm{b}} \leq \boldsymbol{r}_{0}$, then $J_{a}=\infty$.

The total cost of the path radar threat is expressed as:

$$
J_{i j}^{1}=\sum_{a=1}^{N} J_{a}
$$

In formula: $N$ represents the number of voyage segments, $J_{a}$ represents the radar threat cost in the $a$-th road.

3.3 The function of voyage total cost. When our UAVs attack the ground target of enemy, the function of voyage cost is expressed as:

$$
W=\lambda H+(1-\lambda) J
$$

In formula: $H$ is the fuel cost, $J$ is the cost of radar threat, $\lambda$ is the weight coefficient. 


\section{The application of RRT algorithm in path planning}

RRT algorithm is constructed with a special kind of incremental configuration, making the initial point of state space as the root node, and random extension tree is generated by gradually increasing the leaf nodes. The algorithm can search high-dimensional space quickly and efficiently according to the current environment, and guide the search to blank area based on the random sampling point of state space, which is suitable for solving the path planning problem of geometric constraint and dynamic constraint, the extension of RRT algorithm is shown in fig 2. At present, it has been used in aircraft path planning ${ }^{[5]}$ and mobile robot path planning ${ }^{[6]}$. So when UAV meets moved target, a local path is got by RRT algorithm to achieve accurate strike.

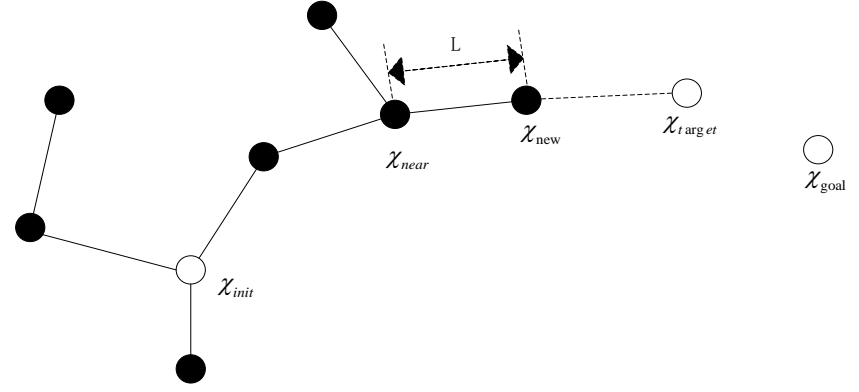

Fig 2 The extension of RRT algorithm

\section{Simulation}

At first, the known threat point, the starting point of the aircraft and the initial target points are given. The Voronoi diagram is constructed for the threat point as the generant, and each side of the threat cost is calculated, which the starting point of UAV is $(1500,400)$, the coordinates before target moved is $(2900,3200)$, so the optimal track generated by Voronoi diagram according to the starting point and initial target point is shown in fig 3:

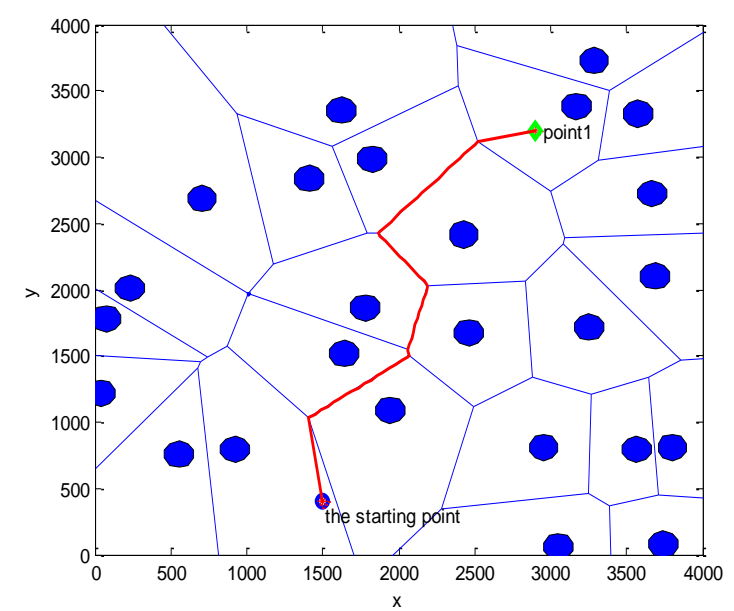

Fig 3. Planning the feasible path according to the starting point and the initial target point

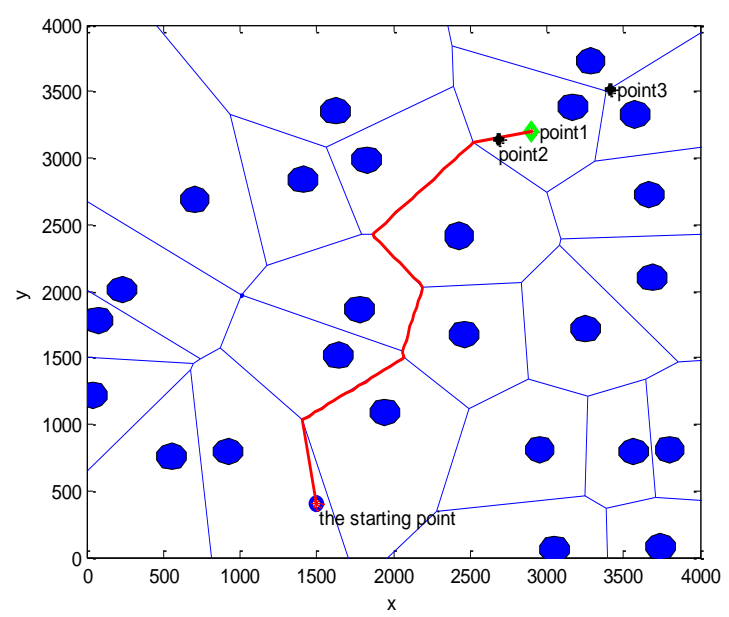

Fig 4. Finding the target point moving and the position after the target point moving

When UAV flying to the position coordinates of points $(2549,3121)$ along the initial path, the original target point location detected according to the airborne detection equipment had been shifting from the points $(2900,3200)$ to the points $(3492,3428)$. As shown in fig 4:

If you took measures in detecting the target moved, a fast search algorithm is used to fast planning a local path to achieve accurately hit for the target, RRT algorithm has a good quickness. So when UAV meets moved target, let the location of finding moved target and the new location of target respectively as the start point and end point of RRT algorithm, then using RRT algorithm gets a local path and achieves accurate strike. Therefore, the whole path is composed of two parts, from start point to the location of finding target moved, the path of this part is part of global path planned by Voronoi 
diagram; from the location of finding target moved to the new location of target, the path of this part is planned by RRT diagram when UAV meets target moved. The overall path planning is shown in fig 5 .

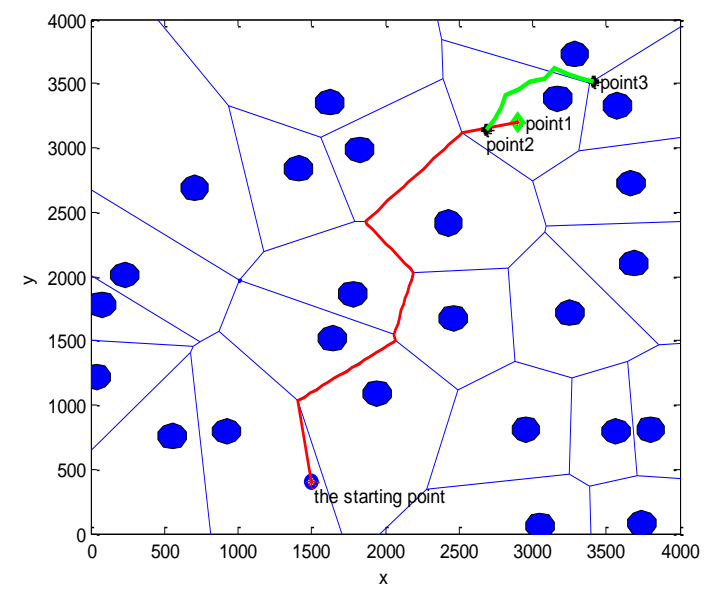

Fig 5. Feasible tracks of online planning after target point moving

\section{Conclusion}

In this paper, the problem of UAV path planning under the environment of moved target is studied. The Voronoi diagram based on threat field is established according to ground threat type. The path planning method adopting Voronoi diagram for initial target point is used to generating the optimal path. And when the target moved, the path originally planned may not meet the requirements, the fast online path planning is needed. In this paper, RRT algorithms is used to plan local path under the environment of moved target. The simulation results show that the combination of the two algorithms not only can plan the best path, but also can adjust the path in time and strike target effectively when the target moved, so as to meet the real-time requirements of the optimal path planning problem.

\section{Acknowledgment}

The research work reported in this paper is supported by the National Nature Science Foundation of China (No. 61503255), Technology Innovation Foundation of Shenyang (No. 14042200 \& No. 14231129).

\section{References}

[1] Chen Xia, Liu Dong, Tang Ting. Path Planning for UCAV in Dynamic and Uncertain Environment Based on Focused D*Algorithm[C]. 2011 Fourth International Symposium on Computational Intelligence and Design (ISCID), 2011: 55-58.

[2] FENG Lin, JIA Jing-hui. Improved algorithm of RRT path planning based on comparison optimization [J]. Computer Engineering and Applications, 2011, 47(3): 210-213+228.

[3] TAN Ruo-lin. RESEARCH AND IMPLEMENTATION OF MULTI-UAV REAL-TIME PATH PLANNING BASED ON MULTI-AGENT SYSTEM [D], University of Electronic Science and Technology of China, 2013.

[4] Wang D B, Wang Y, Jiang W Y and Pu H Z, "Unmanned aerial vehicles cooperative path planning for ground target tracking via chemical reaction optimization”, 123 Sin Tech, vol. 45, (2015), pp. 583-594

[5] Peng H, Wang L, Shen L C. The Modified RRT-based Real-Time Route Planning for UAV Area Target Searching. JOURNAL OF NATIONAL UNIVERSITY OF DEFENSE TECHNOLOGY 2009, 31(5): 86-91. 
[6] Fan X P, Li S Y. Algorithm of Dynamic Motion Planning for Wheeled Mobile Robot whit Kinematic Nonholonomic Constraints [J]. Control and Decision, 2005, 20(7):786-788. 